\title{
Editorial
}

\section{Advances in Molecular Genetics and the Molecular Biology of Deafness}

\author{
Shin-ya Nishio, ${ }^{1}$ Isabelle Schrauwen, ${ }^{2}$ Hideaki Moteki, ${ }^{1}$ and Hela Azaiez ${ }^{3}$ \\ ${ }^{1}$ Shinshu University School of Medicine, Matsumoto, Nagano 390-8621, Japan \\ ${ }^{2}$ Translational Genomics Research Institute, Phoenix, AZ 85004, USA \\ ${ }^{3}$ Department of Otolaryngology, University of Iowa, Iowa City, IA 52246, USA \\ Correspondence should be addressed to Shin-ya Nishio; nishio@shinshu-u.ac.jp
}

Received 9 June 2016; Accepted 13 June 2016

Copyright (C) 2016 Shin-ya Nishio et al. This is an open access article distributed under the Creative Commons Attribution License, which permits unrestricted use, distribution, and reproduction in any medium, provided the original work is properly cited.

Congenital sensorineural hearing loss is the most common sensory disorder, with approximately 1 in every 1000 newborns in developed countries suffering from severeto-profound hearing loss. At least half of those cases are attributable to genetic causes with more than 90 causative genes identified to date reflecting the complex clinical and genetic landscapes of hereditary hearing loss $[1,2]$.

Recent advances in molecular genetics technologies, notably next-generation sequencing (NGS), have drastically accelerated the identification of novel genes involved in hearing mechanism and expanded the mutational spectrum of known deafness-causing genes [3-9]. In addition to NGS, recent progress in genome editing, embryonic stem cells, and induced pluripotent stem cells has opened a new gate to a fast and thorough characterization and understanding of the precise functions and mechanisms involved in the biology of hearing and deafness.

This special issue is to exhibit the advances and recent progress in the fields of molecular genetics and molecular biology of hearing and deafness.

The review paper by S. Kitajiri and T. Katsuno (Kyoto University) summarized the importance of the tricellular tight junction proteins (tricellulin, occludin, ILDR1, and the angulin family) in the inner ear by acting as a barrier separating the endolymphatic and perilymphatic spaces, which is essential for the generation and maintenance of the endocochlear potential.

M. Hosoya et al. described the cochlea distribution patterns of KIAA1199 proteins in a nonhuman primate, the common marmoset (Callithrix jacchus). KIAA1199 has been reported as a cause of progressive hearing loss, but its spatial expression showed different and distinct patterns in mouse and rat cochlea. In this report, M. Hosoya at al. showed a more widespread KIAA1199 protein expression in the marmoset. These results are of importance for further investigation and elucidation of the functional role of KIAA1199 in primate cochlea.

Developing novel diagnostic tools that are tailored to specific ethnicities is crucial for a cost-effective genetic screening for deafness. In this issue, F. Zhang et al. described their multiplex genetic screening system "SNPscan assay" used to screen a total of 115 known mutations in GJB2, SLC26A4, and mtDNA 12SrRNA.

Cochlear implantation (CI) is the most important and effective treatment for patients with profound sensorineural hearing loss. However, outcomes vary among patients due to several reasons, one being the heterogeneous nature of the clinical as well as genetic etiology of hearing loss. $\mathrm{H}$. Koyama et al. reported the CI outcomes in five patients with Waardenburg syndrome. They showed that CI is a good and suitable treatment option for Waardenburg syndrome cases.

Statins are inhibitors of the 3-hydroxy-3-methylglutarylcoenzyme A reductase and widely used as cholesterollowering drugs. However, in the last decade, conflicting data about the effect of statins on neuronal cells and the auditory system has been published. K. Leitmeyer et al. studied the effect of simvastatin on spiral ganglion neurons explants in 
vitro and showed its neurotoxic effect that seems to be at least partially mediated by the mevalonate pathway.

In this special issue, we collected both basic and clinical original research articles stimulating the continuing efforts to understand the mechanisms of deafness and hearing systems. It is our wish to increase interest in this field and further accelerate future treatment options based on solid basic research.

\title{
Acknowledgments
}

Finally, as guest editors, we thank all authors, the editors, and reviewers who have contributed to this special issue.

\author{
Shin-ya Nishio \\ Isabelle Schrauwen \\ Hideaki Moteki \\ Hela Azaiez
}

\section{References}

[1] C. C. Morton and W. E. Nance, "Newborn hearing screeninga silent revolution," The New England Journal of Medicine, vol. 354, no. 20, pp. 2151-2164, 2006.

[2] G. Van Camp and R. J. H. Smith, "Hereditary Hearing loss Homepage," http://hereditaryhearingloss.org.

[3] A. E. Shearer, A. P. DeLuca, M. S. Hildebrand et al., "Comprehensive genetic testing for hereditary hearing loss using massively parallel sequencing," Proceedings of the National Academy of Sciences of the United States of America, vol. 107, no. 49, pp. 21104-21109, 2010.

[4] T. Walsh, H. Shahin, T. Elkan-Miller et al., "Whole exome sequencing and homozygosity mapping identify mutation in the cell polarity protein GPSM2 as the cause of nonsyndromic hearing loss DFNB82," American Journal of Human Genetics, vol. 87, no. 1, pp. 90-94, 2010.

[5] Z. Brownstein, L. M. Friedman, H. Shahin et al., "Targeted genomic capture and massively parallel sequencing to identify genes for hereditary hearing loss in middle eastern families," Genome Biology, vol. 12, no. 9, article R89, 2011.

[6] S. De Keulenaer, J. Hellemans, S. Lefever et al., "Molecular diagnostics for congenital hearing loss including 15 deafness genes using a next generation sequencing platform," $B M C$ Medical Genomics, vol. 5, article 17, 2012.

[7] I. Schrauwen, M. Sommen, J. J. Corneveaux et al., "A sensitive and specific diagnostic test for hearing loss using a microdroplet PCR-based approach and next generation sequencing," American Journal of Medical Genetics A, vol. 161, no. 1, pp. 145-152, 2013.

[8] M. Miyagawa, T. Naito, S.-Y. Nishio, N. Kamatani, and S.-I. Usami, "Targeted exon sequencing successfully discovers rare causative genes and clarifies the molecular epidemiology of japanese deafness patients," PLoS ONE, vol. 8, article e71381, 2013.

[9] S.-Y. Nishio and S.-I. Usami, "Deafness gene variations in a 1,120 nonsyndromic hearing loss cohort: molecular epidemiology and deafness mutation spectrum of patients in Japan," Annals of Otology, Rhinology \& Laryngology, vol. 124, pp. 49S-60S, 2015. 


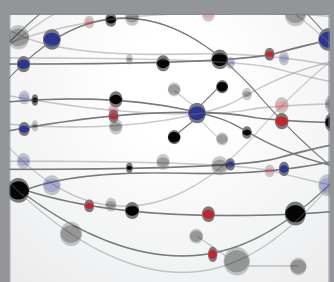

The Scientific World Journal
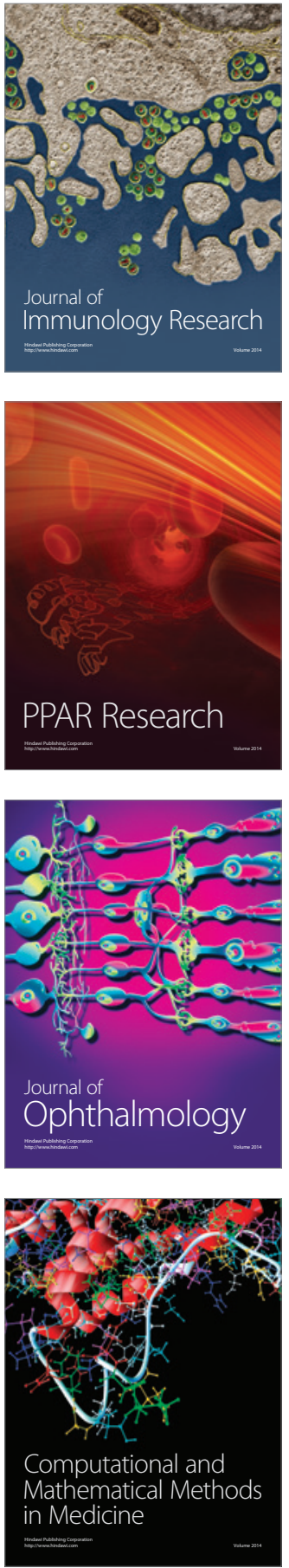

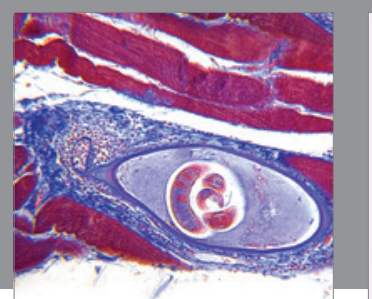

Gastroenterology Research and Practice

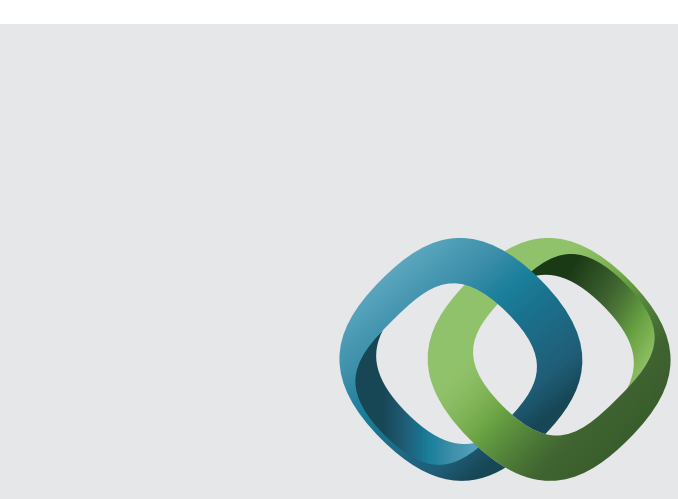

\section{Hindawi}

Submit your manuscripts at

http://www.hindawi.com
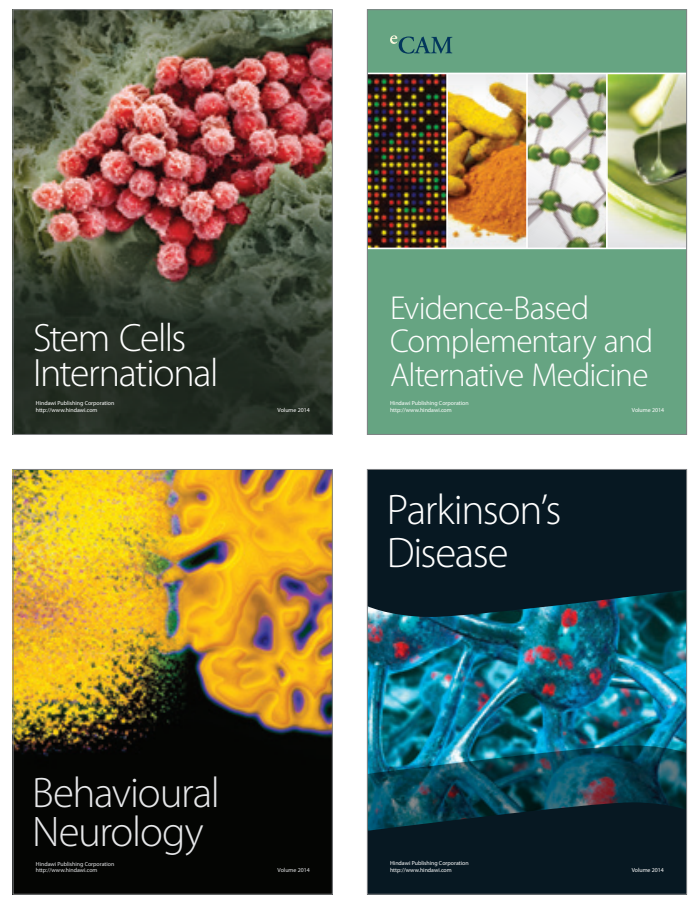
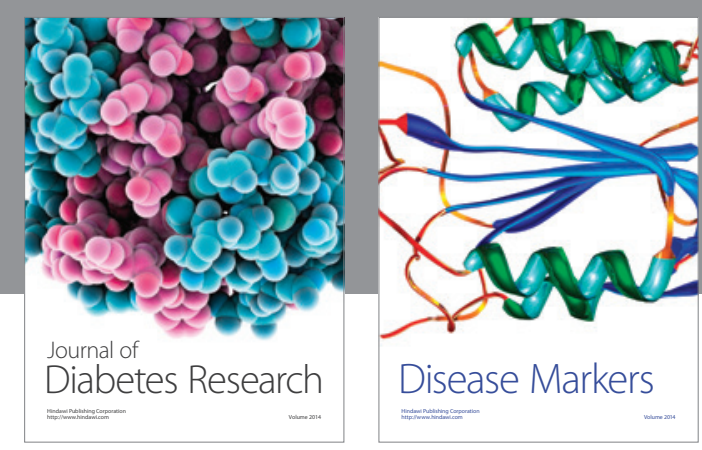

Disease Markers
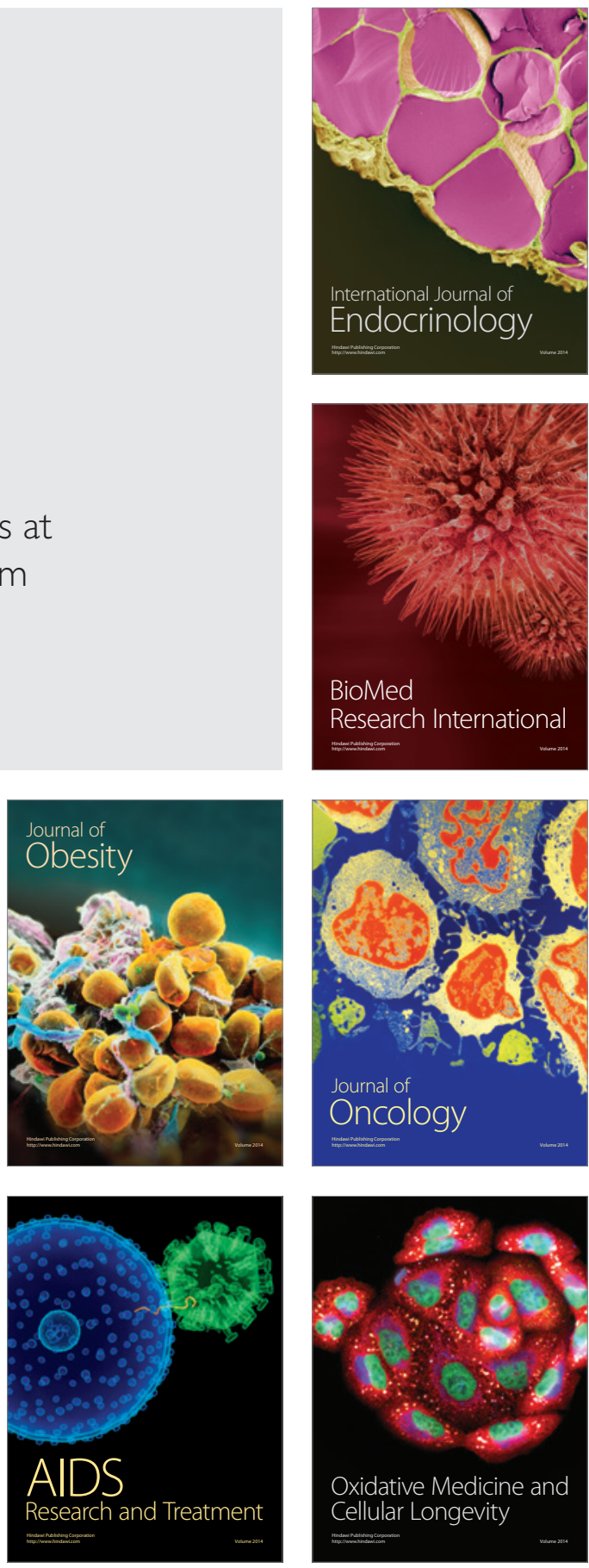